\title{
ANÁLISIS FACIAL FOTOGRÁFICO EN LA ETNIA Saraguro: Análisis de PoWell
}

\author{
Facial photographic analysis at the Saraguro ethnic: Powell's analysis
}

\author{
Karla Johanna Llivigañay Jarro ${ }^{1}$, Diego Esteban Palacios Vivar ${ }^{2}$. \\ 1 Alumna, de la Carrera de Odontología, rotante del programa de investigación, \\ Universidad Católica de Cuenca, Cuenca, Ecuador. \\ ${ }^{2}$ Docente/ investigador, Carrera de Odontología, Universidad Católica de Cuenca, \\ Cirujano Maxilofacial, Cuenca, Ecuador. \\ *llivi.jhois05@gmail.com
}

\begin{abstract}
Resumen
La estética facial es una de las preocupaciones en la población humana desde los inicios de la civilización, debido que la belleza es un sinónimo de salud y perfección para la sociedad. Objetivos: Determinar los valores de perfil facial con fotografía utilizando el análisis de Powell de los adolescentes de la parroquia San Pablo de Tenta del cantón Saraguro-Loja, 2020. Materiales y métodos: Se realizó un estudio observacional descriptivo, transversal, con método cuantitativo. El universo consiste en 120 adolescentes, de las cuales solo 75 cumplieron con los criterios de inclusión. Se utilizó el programa de presentación Microsoft Power Point para realizar los trazos fotográficos. Conclusiones: Se encontró diferencias significativas en el ángulo nasofrontal y mentocervical. El ángulo nasofacial y nasomental cumplieron con el rango establecido por Powell.
\end{abstract}

Palabras clave: Asimetría facial, perfil facial, tejidos blandos, grupos étnicos, belleza.

\begin{abstract}
Facial aesthetics has been one of the concerns in the human population since the beginning of civilization, because beauty is a synonym of health and perfection for society. Aim: Determine the facial profile values with photography using Powell's analysis of adolescents from the San Pablo de Tenta parish in the Saraguro-Loja canton, 2020. Material and methods: A descriptive, cross-sectional observational study was carried out using a quantitative method. A descriptive, cross-sectional observational study was carried out with a quantitative method. The universe consists of 120 adolescents, of which only 75 met the inclusion criteria. The Microsoft Power Point presentation program was used to make the photographic traces. Conclusions: Significant differences were found in the nasofrontal and mentocervical angle. The nasofacial and nasomental angle complied with the range established by Powell.
\end{abstract}

Key words: Facial asymmetry, facial profile, Soft tissues, ethnic groups, beauty.

\section{Introducción}

La estética facial estudia la belleza de la cara del ser humano desde los inicios de la civilización, la cultura griega es la primera en aplicar las leyes geométricas para lograr armonía y equilibrio de las diferentes proporciones del rostro, provocando sensaciones placenteras al observador. Para los romanos, egipcios, culturas africanas y asiáticas un individuo hermoso y perfecto es aquel ser con características faciales extraordinarias que existe en la tierra. ${ }^{1}{ }^{4}$ En la edad media, desde el siglo IV hasta el renacimiento, Leonardo Da Vinci, diseña la cara de un individuo perfecto utilizando la "proporción divina", mediante el cual se analiza el rostro desde todos los ángulos dándole formula aritmética mágica en la forma y belleza facial obteniendo un equilibriocite facial. ${ }^{1}{ }^{3}{ }^{3}$ Edward Angle, afirmaba que la simetría y el balance del aspecto del rostro del sujeto, así como la forma y la perfección de la boca, depende de una buena relación con las piezas dentarias. Por lo cual es importante realizar la valoración estética mediante la exploración clínica que es realizada por el profesional, y este es el encargado de encontrar rasgos faciales desproporcionados y asimétricos que conllevan a problemas estéticos. ${ }^{5},{ }^{6}$

El triángulo estético de Powell analiza de manera simple las principales masas estéticas del rostro y cuello empleando ángulos relaciones entre sí. Se realiza con trazos de líneas sobre los tejidos blandos para formar ángulos, utilizando 
radiografías o fotografías. ${ }^{7}, 8$

Los planos y ángulos que se utilizan son:

- Plano facial: Línea desde la glabela (Gl) a pogonion (Pg) que forma un ángulo con el plano de Frankfort cuyo rango oscila entre 80 y 95 grados. $^{8}{ }_{-} 11$

- Ángulo nasofrontal: Primera línea desde la glabela a nasión $(\mathrm{N})$, segundo desde la punta del dorso de la nariz hasta nasión, se formara un ángulo cuyo rango normal es entre $115^{\circ}$ a $130^{\circ} .{ }^{12}{ }_{-}{ }^{16}$

- Ángulo nasofacial: Primero, está determinado por le plano facial, luego la línea va desde la punta del dorso de la nariz a nasión que forma un ángulo cuyo rango normal para mujeres es de $30^{\circ}$ y para las hombres de $40^{\circ} .{ }^{12}{ }_{-} 16$

- Ángulo nasomental: Empieza con el trazado de la línea estética de Ricketts (plano E) desde la punta del dorso de la nariz a mentón (Me), luego se traza un línea desde la punta del dorso de la nariz hasta nasión que forma un ángulo cuya norma es de 120 a $132^{\circ} \cdot{ }^{12 \_} 16$

- Ángulo mentocervical: Primero, línea de glabela a pogonion, segundo desde el punto $\mathrm{C}$ a mentón que forma un ángulo cuyo rango normal es de $80^{\circ}$ a $95^{\circ} .12_{-} 16$

Objetivo: Este estudio tiene la finalidad de evaluar el Perfil facial con fotografía utilizando el Análisis de Powell de los adolescentes de la Parroquia San Pablo de Tenta del Cantón Saraguro-Loja, 2020.

\section{Materiales y métodos}

Se realizó un estudio transversal, observacional, descriptivo, con método cuantitativo, con el objetivo de establecer valores de perfil facial con fotografía mediante el análisis de Powell. Se desarrolló en la parroquia San Pablo de Tenta que cuenta con una población de 3.676 habitantes, ubicada a 20 minutos del cantón Saraguro provincia de Loja, el universo estuvo estructurado por 120 adolescentes que corresponden al número de asistentes de la institución educativa, la muestra fue de 75 adolescentes que cumplieron con los criterios de inclusión (45 mujeres y de 30 hombres), la cual fue obtenida mediante el muestro no probabilístico por conveniencia. Los criterios de inclusión decretados fueron los siguientes: De inclusión: Estudiantes de 14 a 19 años matriculados en el período 2019-2020. De exclusión: Estudiantes con uso actual o previo de ortodoncia $\mathrm{u}$ ortopedia, antecedentes de trauma o patología facial, representante legal o estudiantes que no deseaba participar en el estudio.

Para la distribución de la toma fotografía a los estudiantes se le asignó un numero agrupándoles por etnia mestizo e indígena Saraguro de sexo femenino y masculino, se utilizó la cámara digital canon EOS T6 KIT 18-55MM.

Los requisitos fueron: la cabeza debe estar en posición natural, los labios y mandíbula en reposo y relajados. Para que la imagen fuera correcta, se tomó como referencia la pestaña superior del ojo izquierdo. El enfoque fue a nivel del ángulo externo del ojo.

Se seleccionó las fotografías en buenas y malas, utilizando el programa Adobe Photoshop CC versión 14.0, se recortaron las imágenes y se dejó el tamaño real de los rostros manteniendo las dimensiones proporcionales de altura y ancho.

En la figura 1 los valores promedio del análisis de Powell: A) Mestizo femenina: Ángulo nasofronal $137^{\circ}$, nasofacial $30^{\circ}$, nasomental $127^{\circ}$, mentocervical $93^{\circ}$. B) Indígena Saraguro femenina: Ángulo nasofronal $130^{\circ}$, nasofacial $35^{\circ}$, nasomental $122^{\circ}$, mentocervical $95^{\circ}$.

En la figura 2 lo valores promedio del análisis de Powell: A) Mestizo masculino: Ángulo nasofronal $130^{\circ}$, nasofacial $40^{\circ}$, nasomental $122^{\circ}$, mentocervical $121^{\circ}$. B) Indígena Saraguro masculino: Ángulo nasofronal $120^{\circ}$, nasofacial $40^{\circ}$, nasomental $120^{\circ}$, mentocervical $90^{\circ}$. Se realizó los trazos, utilizando el programa de presentación Microsoft Power Point, utilizando una regla y escuadra digital obteniendo el ángulo nasofrontal, nasofacial, nasomental, mentocervical.

Para el procesamiento estadístico, se realizó una base de datos en el programa informático Microsoft Excel. Se utilizó fórmulas matemáticas para obtener los datos del estudio.

\section{Resultados}

En la población estudiada 45 adolescentes pertenecen al sexo femenino en un $60 \%, 30$ adolescentes pertenecen al sexo masculino en un $40 \%$, obteniendo una población de 75 estudiantes en un $100 \%$ (Tabla 1).

Se observa que en la población estudiada el $25 \%$ de las mestizas del sexo femenino presento valores de $140,5^{\circ}$ y el $75 \%$ valores de $147^{\circ}$, comparada con la etnia indígena Saraguro donde el $25 \%$ presento $137,5^{\circ}$ y el $75 \% 144^{\circ}$, observando que los valores se encuentran superiores al rango establecido por Powell. También el $25 \%$ de los mestizos del sexo masculino presento valores de $133,5^{\circ}$ y el $75 \%$ valores de $140^{\circ}$, comparada con la etnia indígena Saraguro donde el $25 \%$ presento $130^{\circ}$ y el $75 \% 140^{\circ}$, observando que los valores se encuentran superiores al rango establecido por Powell (Tabla 2).

Por lo cual se propone una nueva norma estándar para las mestizas del sexo femenino de $140^{\circ}-150^{\circ}$ y para la etnia indígena Saraguro de $140^{\circ}-155^{\circ}$, para los mestizos del sexo masculino de $130^{\circ}-140^{\circ}$ y para la etnia indígena Saraguro de $135^{\circ}-145^{\circ}$ (Tabla 2).

Se observa que en la población estudiada el $25 \%$ de las mestizas del sexo femenino presento valores de $30^{\circ}$ y el $75 \%$ valores de $34,75^{\circ}$, comparada con la etnia indígena Saraguro donde el $25 \%$ presento $35^{\circ}$ y el $75 \% 40^{\circ}$, observando que los valores se encuentran dentro del rango establecido por Powell. También el $25 \%$ de los mestizos del sexo masculino 


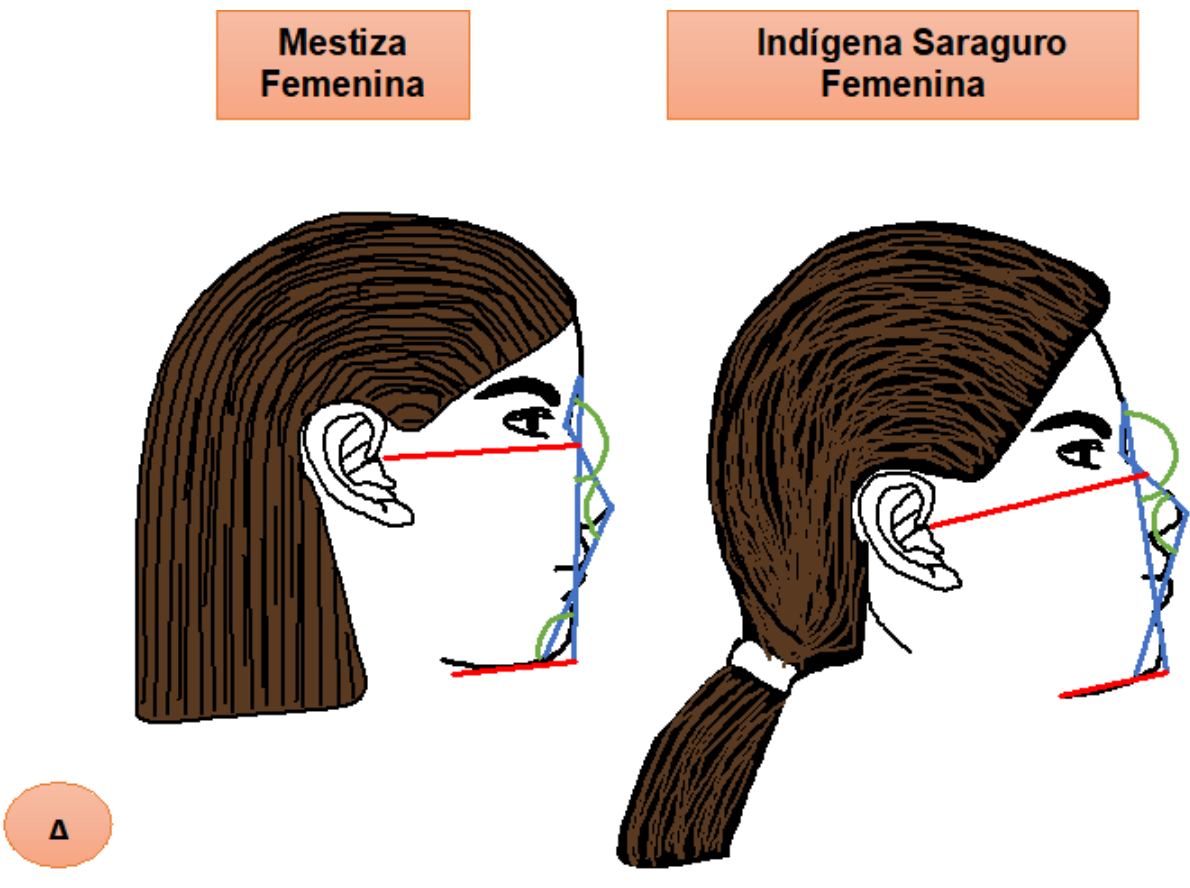

Fig. 1. Valores promedio del análisis de Powell
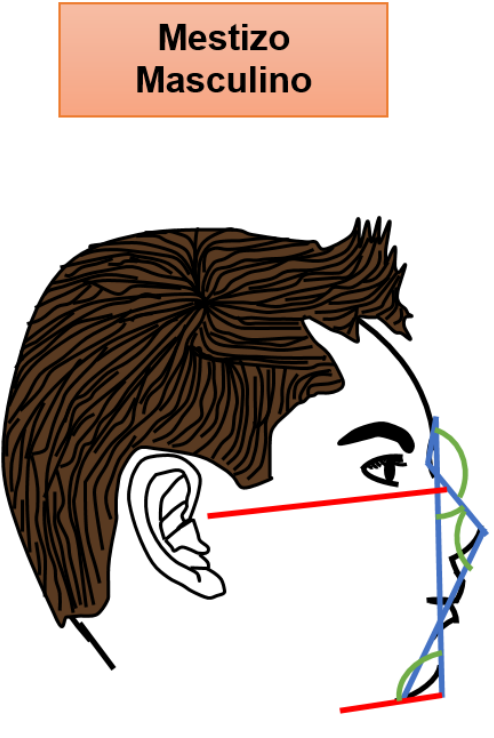

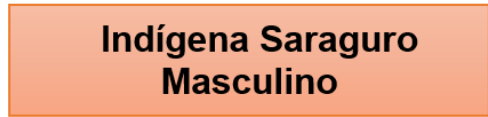
Masculino

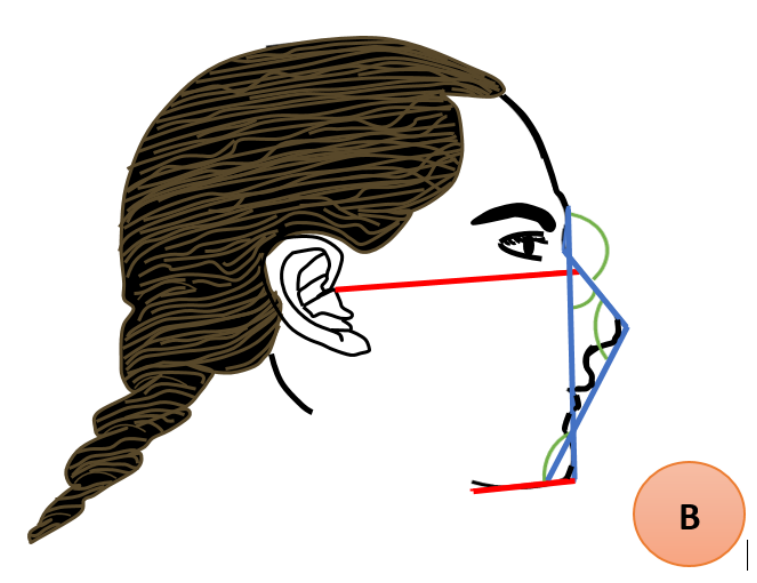

Fig. 2. Figuras del análisis de Powell

presento valores de $31,5^{\circ}$ y el $75 \%$ valores de $39,25^{\circ}$, comparada con la etnia indígena Saraguro donde el $25 \%$ presentó $35^{\circ}$ y el $75 \% 40^{\circ}$, observando que los valores se encuentran dentro del rango establecido por Powell (Tabla 3 ).
Se observa que en la población estudiada el $25 \%$ de las mestizas del sexo femenino presentó valores de $122,25^{\circ}$ y el $75 \%$ valores de $127^{\circ}$, comparada con la etnia indígena Saraguro donde el $25 \%$ presentó $123^{\circ}$ y el $75 \% 128^{\circ}$, 
Tabla N¹ Distribución de población estudiada en relación al sexo y etnia.

\begin{tabular}{|c|c|c|c|c|c|}
\hline \multirow{2}{*}{\multicolumn{2}{|c|}{ Etnia }} & \multicolumn{2}{|c|}{ Sexo } & \multirow{2}{*}{ Total } & \multirow{2}{*}{$\begin{array}{c}\text { Porcentaje } \\
\text { total }\end{array}$} \\
\hline & & Femenino & Masculino & & \\
\hline \multicolumn{2}{|c|}{ Mestizo } & 16 & 12 & 28 & $37 \%$ \\
\hline \multicolumn{2}{|c|}{$\begin{array}{l}\text { Indígena } \\
\text { Saraguro }\end{array}$} & 29 & 18 & 47 & $63 \%$ \\
\hline \multirow[t]{2}{*}{ Total } & $\mathrm{n}$ & 45 & 30 & 75 & $100 \%$ \\
\hline & $\%$ & $60 \%$ & $40 \%$ & $100 \%$ & \\
\hline
\end{tabular}

Tabla $N^{\circ}$ 2. Ángulo Nasofrontal $\left(115-130^{\circ}\right)$.

\begin{tabular}{ccrr}
\hline & \multicolumn{3}{c}{ Sexo } \\
Etnia & & Femenino & Masculino \\
\hline Mestizo & P25\% & $140,5^{\circ}$ & $133,5^{\circ}$ \\
& P50\% & $145^{\circ}$ & $139^{\circ}$ \\
& P75\% & $147^{\circ}$ & $140^{\circ}$ \\
& Rango & $140-150^{\circ}$ & $130-140^{\circ}$ \\
Indígena & P25\% & $137,5^{\circ}$ & $130^{\circ}$ \\
& P50\% & $140^{\circ}$ & $135^{\circ}$ \\
& P75\% & $144^{\circ}$ & $140^{\circ}$ \\
& Rango & $140-155^{\circ}$ & $135-145^{\circ}$ \\
& & & \\
\hline
\end{tabular}

Tabla $\mathrm{N}^{\circ}$ 3. Ángulo Nasofacial $\left(30-40^{\circ}\right)$.

\begin{tabular}{cccr}
\hline \multirow{2}{*}{ Etnia } & \multicolumn{2}{c}{ Sexo } \\
\hline Mestizo & $\mathrm{P} 25 \%$ & $30^{\circ}$ & $31.5^{\circ}$ \\
& $\mathrm{P} 50 \%$ & $30^{\circ}$ & $34.5^{\circ}$ \\
& $\mathrm{P} 75 \%$ & $34,75^{\circ}$ & $39.25^{\circ}$ \\
& $\mathrm{P} 25 \%$ & $35^{\circ}$ & $35^{\circ}$ \\
Saraguro & $35^{\circ}$ & $36^{\circ}$ \\
& $\mathrm{P} 50 \%$ & $40^{\circ}$ & $40^{\circ}$ \\
\hline
\end{tabular}

Tabla $N^{\circ}$ 4. Ángulo Nasomental $\left(120-132^{\circ}\right)$.

\section{Sexo}

\begin{tabular}{lrrr} 
Etnia & & Femenino & Masculino \\
\hline Mestizo & $\mathrm{P} 25 \%$ & $122,25^{\circ}$ & $122^{\circ}$ \\
& $\mathrm{P} 50 \%$ & $125^{\circ}$ & $123^{\circ}$ \\
& $\mathrm{P} 75 \%$ & $127^{\circ}$ & $126,5^{\circ}$ \\
Indígena & $\mathrm{P} 25 \%$ & $123^{\circ}$ & \\
Saraguro & $\mathrm{P} 50 \%$ & $126^{\circ}$ & $120^{\circ}$ \\
& $\mathrm{P} 75 \%$ & $128^{\circ}$ & $127,5^{\circ}$ \\
\hline
\end{tabular}

observando que los valores se encuentran dentro del rango establecido por Powell. También el $25 \%$ de los mestizos del sexo masculino presentó valores de $122^{\circ}$ y el $75 \%$ valores de $126,5^{\circ}$, comparada con la etnia indígena Saraguro donde el $25 \%$ presentó $120^{\circ}$ y el $75 \% 127,5^{\circ}$, observando que los valores se encuentran dentro del rango establecido por Powell (Tabla 4).

Tabla 5. Ángulo Mentocervical $\left(80-95^{\circ}\right)$

\begin{tabular}{lrrr}
\hline & & \multicolumn{2}{c}{ Sexo } \\
Etnia & & Femenino & Masculino \\
\hline Mestizo & P25\% & $93,5^{\circ}$ & $90^{\circ}$ \\
& P50\% & $95^{\circ}$ & $96,5^{\circ}$ \\
& P75\% & $99^{\circ}$ & $104,5^{\circ}$ \\
& Rango & $90-100^{\circ}$ & $90-110^{\circ}$ \\
Indígena & P25\% & $90^{\circ}$ & $91,5^{\circ}$ \\
& P50\% & $90^{\circ}$ & $99^{\circ}$ \\
& P75\% & $95^{\circ}$ & $101,5^{\circ}$ \\
& Rango & $85-95^{\circ}$ & $95-115^{\circ}$ \\
\hline
\end{tabular}

Se observa que en la población estudiada el $25 \%$ de las mestizas del sexo femenino presento valores de $93,5^{\circ}$ y el $75 \%$ valores de $99^{\circ}$, comparada con la etnia indígena Saraguro donde el $25 \%$ presento $90^{\circ}$ y el $75 \% 95^{\circ}$; observando que los valores se encuentran superiores del rango establecido por Powell. También el $25 \%$ de los mestizos del sexo masculino presento valores de $90^{\circ}$ y el $75 \%$ valores de $104,5^{\circ}$; comparada con la etnia indígena Saraguro donde el $25 \%$ presentó $91,5^{\circ}$ y el $75 \% 101,5^{\circ}$; observando que los valores se encuentran superiores al rango establecido por Powell (Tabla 5).

Por lo cual se propone una nueva norma estándar para las mestizas del sexo femenino de $90^{\circ}-100^{\circ}$ y para la etnia indígena Saraguro de $85^{\circ}-95^{\circ}$; para los mestizos del sexo masculino de $90^{\circ}-110^{\circ}$ y para la etnia indígena Saraguro de $95^{\circ}-115^{\circ}$ (Tabla 5).

\section{Discusión}

El análisis de Powell analiza de manera sencilla y práctica las principales masas estéticas del rostro, utilizando ángulos interrelacionados. La mayoría de la población presenta cierta preocupación por tener un rostro perfecto. Un examen clínico adecuado es el primer paso para valorar los tejidos blandos, esqueléticos, distinguiendo el rostro del individuo, su forma y las proporciones para reconocer cualquier anomalía y comprobar si esto afecta el equilibrio facial. Se debe tomar en cuenta que existe muchos aspectos que pueden afectar la interpretación y un buen resultado: Edad, raza, sexo, hábitos corporales y la personalidad de 
individuo. Si existen alteraciones en las proporciones del rostro, estas pueden ser modificables con tratamiento de ortodoncia o cirugía maxilofacial.

El ángulo nasofrontal en la etnia Mochica de Perú, la etnia indígena de la comunidad sierra Cacha de Ecuador, la etnia uro de la comunidad de la sierra de los Uros en Perú, obtuvieron valores superiores al rango establecido por Powell, lo cual se establece que no existe diferencias significativas con los valores obtenidos en mestizos y la etnia indígena Saraguro del presente estudio. ${ }^{12-14}$

El ángulo nasofacial en la etnia Mochica de Perú, la etnia indígena de la comunidad sierra Cacha de Ecuador, la etnia uro de la comunidad de la sierra de los Uros en Perú, obtuvieron valores dentro al rango establecido por Powell, lo cual se establece que no existe diferencias significativas con los valores obtenidos en mestizos y etnia indígena Saraguro del presente estudio. ${ }^{12-14}$

El ángulo nasomental en la etnia Mochica de Perú, la etnia uro de la comunidad de la sierra de los Uros en Perú, obtuvieron valores dentro del rango establecido por Powell, por lo cual se establece que no existe diferencias significativas de los valores obtenidos en mestizos y la etnia indígena Saraguro del presente estudio, pero la etnia indígena de la comunidad sierra Cacha de Ecuador obtuvo valores superiores, lo cual demuestra que existe diferencias significativas con los mestizos y la etnia indígena Saraguro del presente estudio. ${ }^{12-14}$

El ángulo mentocervical en la etnia indígena de la comunidad sierra Cacha de Ecuador, la etnia uro de la comunidad de la sierra de los Uros en Perú, obtuvieron valores superiores al rango establecido por Powell, lo cual se establece que no existe diferencias significativas con los valores obtenidos en mestizos y la etnia indígena Saraguro del presente estudio, pero la etnia Mochica de Perú obtuvo valores dentro del rango establecido por Powell, lo cual demuestra que existe diferencias significativas con los mestizos y la etnia indígena Saraguro del presente estudio. ${ }^{12-14}$

\section{Conclusiones}

- Los valores de los ángulos nasofacial, nasomental se encuentran dentro del rango establecido por Powell, siendo aplicable en la etnia mestiza e indígena Saraguro.

- Los valores del ángulo nasofrontal difieren de manera importante del rango establecido por Powell, por lo que recomendamos considerar un nuevo rango para la etnia mestiza del sexo femenino de $140^{\circ}-150^{\circ}$ y para el sexo masculino de $130^{\circ}-140^{\circ}$, para la etnia indígena Saraguro del sexo femenino de $140^{\circ}-155^{\circ}$ y para el sexo masculino de $135^{\circ}-145^{\circ}$.
- Los valores del ángulo mentocervical difieren importantemente del rango establecido por Powell, por lo que recomendamos considerar un nuevo rango para la etnia mestiza del sexo femenino de $90^{\circ}-100^{\circ}$ y para sexo masculino de $90^{\circ}-110^{\circ}$, para la etnia indígena Saraguro del sexo femenino de $85^{\circ}-95^{\circ}$ y para el sexo masculino de $95^{\circ}-115^{\circ}$.

Conflicto de intereses: Los autores del presente estudio manifiestan que no existe ningún conflicto de intereses en relación al tema de estudio.

Agradecimientos : Los autores agradecen a los adolescentes de la Unidad Educativa Bilingüe ABC por participar en el presente estudio.

\section{Referencias}

1 Rodríguez M, Barbería E, Duran J, Muñoz, Vera V. Evolución historia de los conceptos de belleza facial. Revista de Ortodoncia Clínica. 2000; 3(3):156-163.

2 Carrera E, Piña M, Sáez G, Flores A. Valoración estética en pacientes clase III antes y después del tratamiento quirúrgico de acuerdo con la proporción divina. Revista Mexica en Odontología. 2015; 3(3):154-161.

3 Cazar M, Abril M, Recendez N. Análisis estético dentofacial: Base de la terapéutica de las deformidades faciales. Revista OACTIVA UC Cuenca. 2017; 2(3):27-34.

4 Guzmán M, Vera ME, Flores A. Percepción de la estética de la sonrisa por odontólogos Especialistas y pacientes. Revista Mexica en Odontología. 2015; 3(1):13-21.

5 Mendoza M. Análisis facial en Ortodoncia. Revista Kiru. 2004; 1(1):48-50.

6 García S, Orrego G. Parámetros estéticos de perfil facial. Revista Kiru. 2014; 11(1):86-90.

7 García M, Chacón CE, Medina X. Percepción de los parámetros de estética facial en los perfiles recto, convexo y cóncavo, en caras largas, media y corta. Revista Odontológica Colegial. 2010; 6(6): 6-53.

8 Perez L, Kú Y, Colóme G, Santana A. Correlación de Perfil y Arcos dentarios en una población de Yucatán. Revista Mexica de Ortodoncia. 2016; 4(2): 84-87.

9 Huentequeo C, Navarro P, Vásquez O. Análisis Facial, Dentario y Radiográfico de la Normalidad Facial: Estudio Piloto en 29 Mujeres. Int. J. Morphol. 2013; 31(1): 150-155. 10 Puigdollers A. Análisis estético y funcional de los tejidos blandos. Revista de Revistas. 2000; 30:182-188.

11 Ramírez V. Orígenes Históricos de la Ortopedia Maxilofacial. En:Vellini F, Editor. Ortodoncia, Diagnostico y Planificación Clínica. Segunda Edición. Brasil: Editorial Artes Medicas, 2004.p. 19-27.

12 Díaz D. Perfil facial según el Análisis de Powell en personas del centro poblado cruz de Médano del distrito de MórropeLambayeque, 2017 (tesis para la obtención de título de cirujano dentista). Mórrope-Lambayeque. Perú. Universidad Alas Peruanas. 2018. 
13 Carrillo D. Valoración fotográfica de los ángulos faciales en estudiantes de la comunidad de Cacha mediante el análisis de Powell período Septiembre 2016 - Junio 2017 (tesis para la obtención de titulación de odontólogo). Quito. Universidad Central del Ecuador. 2017.

14 Alarcón J. Perfil facial de pobladores peruanos de la Comunidad de los Eros mediante el análisis de Powell (tesis para la obtención de titulación de odontólogo). Lima. Universidad Nacional Mayor de San Marcos. 2003.

15 Toala A. Ángulos de perfil facial frente a parámetros estándar en análisis fotométrico en estudiantes de 17-18 años del Colegio Amazonas. estudio comparativo (tesis para la obtención de titulación de odontólogo). Quito. Universidad Central del Ecuador. 2016.

16 Vásquez, Aurarose. Çomparación de los ángulos del perfil facial entre la norma de Powell y los valores hallados en los pobladores de Paredones Alto, Mórrope"(Tesis para la obtención de titulación de odontólogo). Pimentel. Universidad Señor de Sipán. Pimentel. 2017.

Recibido: 11 de septiembre de 2020

Aceptado: 12 de noviembre de 2020 